\title{
Nonperturbative effective-field theory for two-leg antiferromagnetic spin ladders
}

\author{
D. C. Cabra, ${ }^{1,2}$ A. Dobry, ${ }^{3}$ and G. L. Rossini ${ }^{1}$ \\ ${ }^{1}$ Departamento de Física, Universidad Nacional de la Plata, C.C. 67, (1900) La Plata, Argentina \\ ${ }^{2}$ Facultad de Ingeniería, Universidad Nacional de Lomas de Zamora, Cno. de Cintura y Juan XXIII, \\ (1832) Lomas de Zamora, Argentina \\ ${ }^{3}$ Departamento de Física, Facultad de Ciencias Exactas, Ingeniería y Agrimensura and IFIR (CONICET-UNR) \\ Av. Pellegrini 250, 2000 Rosario, Argentina
}

(Received 27 October 2000; published 19 March 2001)

\begin{abstract}
We study the long wavelength limit of a spin- $\frac{1}{2}$ Heisenberg antiferromagnetic two-leg ladder, treating the interchain coupling in a nonperturbative way. We perform a mean field analysis and then include the fluctuations in an exact way. This allows for a discussion of the phase diagram of the system and provides an effective-field theory for the low-energy excitations. The coset fermionic Lagrangian obtained corresponds to a perturbed $S U(4)_{1} / U(1)$ conformal field theory (CFT). This effective theory is naturally embedded in a $S U(2)_{2} \times Z_{2}$ CFT, where perturbations are easily identified in terms of conformal operators in the two sectors. Crossed and zigzag ladders are also discussed using the same approach.
\end{abstract}

DOI: 10.1103/PhysRevB.63.144408

PACS number(s): 75.50.Ee, 75.10.Jm

\section{INTRODUCTION}

Antiferromagnetic Heisenberg spin ladders have been a subject of central interest during the last years. These are intermediate systems between the gapless critical spin $-\frac{1}{2}$ Heisenberg chain and the ordered spin- $\frac{1}{2}$ two-dimensional system relevant for undoped cuprate superconductors. The simplest realization, i.e., the two-leg ladder, shows a dramatically different excitation spectrum with respect to one of an isolated chain. It has a finite gap to the first excitation and magnetic correlations are short range. Several inorganic compounds have been recently synthesized and modeled as Heisenberg ladders. ${ }^{1}$ Exponential decay of the lowtemperature magnetic susceptibility was the first signal of the existence of a spin gap in two-leg ladder materials. Neutron and optical measurements also manifest the presence of a gap and are consistently described by a two-leg ladder model with exchange integrals of the same order in the chains direction $(J)$ and along the rungs $\left(J^{\prime}\right)$.

Theoretically, the existence of a gap was predicted early from numerical exact diagonalization and strong coupling perturbation theory $\left(J / J^{\prime} \ll 1\right){ }^{2}$ More recently fieldtheoretical techniques have been used to analyze the excitation spectrum in the weak-coupling regime $\left(J^{\prime} / J \ll 1\right)$. 3,4 These treatments give access to the whole low energy excitation spectrum as well as to the dynamical susceptibilities that are essential to comparing with experimental probes. The philosophy underlying this study is the following: spin operators are expressed in the well-known bosonized representation of each chain and the interchain coupling is treated as a small perturbation in this representation. The applicability of these studies is then valid in principle only in the weak-coupling regime and its use in the description of, e.g., the experimentally realized two-leg ladders in which $J^{\prime}$ $\sim J / 2$ should be taken with some care. It is therefore not clear up to which value of $J^{\prime} / J$ the results of Refs. 3 and 4 are applicable, and it is important to develop theoretical methods that could be used beyond the weak coupling regime.
The picture that emerges from the weak-coupling analysis leads to a description in terms of a triplet of massive Majorana fermions and a singlet Majorana fermion with a different mass (which has been estimated to be minus three times the triplet mass). ${ }^{4}$ The only interactions between these fermions are marginal current-current terms, which have been argued to simply renormalize their masses and velocities. A question that has risen in recent studies of the Ramanscattering spectrum, ${ }^{5}$ is whether marginal interactions can in fact be disregarded. In particular, correlation functions obtained disregarding marginal interactions apparently do not fit experiments (see, e.g., Ref. 6).

In this work we analyze the complete phase diagram of the two-leg antiferromagnetic ladder. Our approach, first used here for spin ladders, starts from a fermionic representation of the spin operators in the functional-integral framework, as introduced in Refs. 7-9 for spin chains. With a simple ansatz to the mean-field (MF) configurations we show that the system undergoes a crossover from a weak- to a strong-coupling regime at an intermediate value of $J^{\prime} / J$. We then introduce fluctuations around MF and take them into account at all orders to construct the low-energy effectivefield theory.

The resulting theory corresponds to a coset conformal field theory (CFT) of symmetry $S U(4)_{1} / U(1)_{i s o}$, perturbed by relevant operators (of dimension 1) and marginal operators (of dimension 2) arising from the single occupancy constraint as well as from the amplitude fluctuations of the link fields introduced to decouple the fermionic interactions. It should be noted that our approach is based on the assumption that the local single occupancy constraint can be implemented as a very last step, while it is taken into account globally from the beginning. The correctness of this procedure is not guaranteed from first principles, but is supported a posteriori.

We show that the complete structure of these perturbations can be retained and that they take a simple form in the language of conformal embeddings. In particular, the marginal terms that arise can be easily classified in the new 
language and their effect can then be studied in a nonperturbative way. When the relevant perturbations are expressed in the embedded $S U(2)_{2} \times Z_{2}$ CFT language the spectrum is naturally separated in the triplet and singlet of Majorana fermions. These results, which are valid up to $J^{\prime} / J \approx 8 / \pi^{2}$, extend to finite coupling the weak-coupling study of Ref. 4. It should be stressed that recent estimates of the ratio of exchange constants lead to values of $J^{\prime} / J$ around $\frac{1}{2}$ in several cuprate materials. ${ }^{10}$

In order to illustrate the generality and ease of use of our approach, it is then applied to the so-called crossed ladders and zigzag ladders. Phase diagrams and low-energy theories are obtained in the region containing the weak coupling limit; further analysis and details will be considered elsewhere.

The paper is organized as follows. In Sec. II we introduce the model, present Hubbard-Stratonovich decoupling techniques, and perform a MF analysis, discussing the resulting phase diagram. In Sec. III we construct the low energy effective-field theory: our theory contains four Dirac fermionic species corresponding to the spin and band indices of the ladder. In Sec. IV we show that the theory has a natural relation to $S U(2)_{2} \times Z_{2}$ CFT through conformal embedding (the last part arises from the two electronic bands). In Sec. V we briefly report results on crossed and zigzag ladders. Finally, in Sec. VI the conclusions and possible further developments of our method are given.

\section{MEAN-FIELD ANALYSIS}

We consider the Heisenberg Hamiltonian for a two-leg spin- $-\frac{1}{2}$ ladder,

$$
H=\sum_{n=1}^{N} \sum_{l=1}^{2}\left(J \vec{S}_{n}^{(l)} \cdot \vec{S}_{n+1}^{(l)}+\frac{J^{\prime}}{2} \vec{S}_{n}^{(l)} \cdot \vec{S}_{n}^{(l+1)}\right),
$$

where $N$ is the number of sites along the chains, and $J>0$ and $J^{\prime}>0$ are the couplings between adjacent spins along the legs and rungs respectively. For mathematical convenience we assume periodic boundary conditions in both directions (notice that the Hamiltonian is suitably written for arbitrary $n$-leg ladders; in the present case the physical coupling along the rungs is in effect $J^{\prime}$ ).

The spin variables can be represented in terms of fermionic operators with $\operatorname{spin} c_{n, \alpha}^{(l)}$ as

$$
\vec{S}_{n}^{(l)}=c_{n, \alpha}^{\dagger(l)} \frac{\vec{\sigma}_{\alpha \beta}}{2} c_{n, \beta}^{(l)},
$$

where $\vec{\sigma}$ are Pauli matrices, together with a local constraint that ensures one spin per site, imposed on the physical states by

$$
c_{n, \alpha}^{\dagger(l)} c_{n, \alpha}^{(l)}|p h y s\rangle=|p h y s\rangle .
$$

Throughout this paper we will not use the summation convention for neither site nor leg indices; repeated spin (Greek) indices are summed.

Using Eqs. (2) and (3) the Hamiltonian (1) can be rewritten as

$$
\begin{aligned}
H= & -\sum_{n=1}^{N} \sum_{l=1}^{2}\left(\frac{J}{2} c_{n, \alpha}^{\dagger(l)} c_{n+1, \alpha}^{(l)} c_{n+1, \beta}^{\dagger(l)} c_{n, \beta}^{(l)}\right. \\
& \left.+\frac{J^{\prime}}{4} c_{n, \alpha}^{\dagger(l)} c_{n, \alpha}^{(l+1)} c_{n, \beta}^{\dagger(l+1)} c_{n, \beta}^{(l)}\right)+C,
\end{aligned}
$$

where $C=-N / 2$ is an irrelevant constant term.

We now trade Eq. (4) for a quadratic Hamiltonian via a Hubbard-Stratonovich transformation, at the usual price of introducing auxiliary fields $B_{n}^{(l)}$ associated to terms containing $c_{n+1, \alpha}^{\dagger(l)} c_{n, \alpha}^{(l)}$, and $B_{n}^{\prime}$ associated to terms containing $c_{n, \beta}^{\dagger(l+1)} c_{n, \beta}^{(l)}$. It is natural to interpret $B_{n}^{(l)}$ as localized on the leg $(l)$ links between sites $n$ and $n+1$, and $B_{n}^{\prime}$ as localized on the rung links. After the transformation the Hamiltonian reads

$$
\begin{aligned}
H= & \frac{J}{2} \sum_{n=1}^{N} \sum_{l=1}^{2}\left(B_{n}^{(l)} c_{n, \alpha}^{\dagger(l)} c_{n+1, \alpha}^{(l)}+B_{n}^{\dagger(l)} c_{n+1, \beta}^{\dagger(l)} c_{n, \beta}^{(l)}+B_{n}^{\dagger(l)} B_{n}^{(l)}\right) \\
& +\frac{J^{\prime}}{4} \sum_{n=1}^{N} \sum_{l=1}^{2}\left(B_{n}^{\prime} c_{n, \alpha}^{\dagger(l)} c_{n, \alpha}^{(l+1)}+B_{n}^{\dagger^{\prime}} c_{n, \beta}^{\dagger(l+1)} c_{n, \beta}^{(l)}+B_{n}^{\dagger^{\prime}} B_{n}^{\prime}\right) .
\end{aligned}
$$

As we look for a low-energy effective theory, we treat the $B$ variables in a long-wave approximation. To this end, we parametrize these fields in terms of real MF values $\left(B_{0}, B_{0}^{\prime}\right)$ and fluctuations

$$
B_{n}^{(l)}=B_{0} \exp \left(i a A_{n}^{(l)}+a R_{n}^{(l)}\right), \quad B_{n}^{\prime}=B_{0}^{\prime} \exp \left(i a A_{n}^{\prime}+a R_{n}^{\prime}\right) .
$$

Notice that we have included both phase and amplitude fluctuations, which will play important different roles in the following. For this reason, we explicitly distinguish the Hermitian $\left(R_{n}^{(l)}, R_{n}^{\prime(l)}\right)$ and anti-Hermitian $\left(i A_{n}^{(l)}, i A_{n}^{\prime(l)}\right)$ parts of the fluctuation fields. The expression for $B_{n}^{\prime}$ will be eventually modified when $B_{0}^{\prime}=0$ [see Eq. (31)].

As a first step, we perform the MF evaluation of the Hamiltonian (5) by setting the fluctuations to zero. The resulting MF Hamiltonian is then a tight-binding model for two coupled chains,

$$
\begin{aligned}
H_{m f}= & -t \sum_{n=1}^{N} \sum_{l=1}^{2}\left(c_{n, \alpha}^{\dagger(l)} c_{n+1, \alpha}^{(l)}+c_{n+1, \beta}^{\dagger(l)} c_{n, \beta}^{(l)}\right) \\
& -2 t^{\prime} \sum_{n=1}^{N}\left(c_{n, \alpha}^{\dagger(1)} c_{n, \alpha}^{(2)}+c_{n, \beta}^{\dagger(2)} c_{n, \beta}^{(1)}\right)+\frac{4 N}{J} t^{2}+\frac{8 N}{J^{\prime}} t^{\prime 2},
\end{aligned}
$$

where

$$
t=-\frac{J B_{0}}{2}, \quad t^{\prime}=-\frac{J^{\prime} B_{0}^{\prime}}{4} .
$$

The coupled tight-binding model is easily diagonalized by means of a double Fourier transform. We first decouple two bands by means of 


$$
\begin{aligned}
& c_{n, \alpha}^{(1)}=\frac{1}{\sqrt{2}}\left(c_{n, \alpha}^{(+)}-c_{n, \alpha}^{(-)}\right), \\
& c_{n, \alpha}^{(2)}=\frac{1}{\sqrt{2}}\left(c_{n, \alpha}^{(+)}+c_{n, \alpha}^{(-)}\right)
\end{aligned}
$$

and then introduce pseudomomentum operators $d^{(+)}, d^{(-)}$by

$$
\begin{aligned}
& c_{n, \alpha}^{(+)}=\frac{1}{\sqrt{2 N}} \sum_{m=1}^{N} d_{m, \alpha}^{(+)} \exp \left(-i \frac{2 \pi m n}{N}\right), \\
& c_{n, \alpha}^{(-)}=\frac{1}{\sqrt{2 N}} \sum_{m=1}^{N} d_{m, \alpha}^{(-)} \exp \left(-i \frac{2 \pi m n}{N}\right),
\end{aligned}
$$

in terms of which the Hamiltonian reads

$$
\begin{aligned}
H_{m f}= & -\sum_{m=1}^{N}\left[2 t \cos \left(\frac{2 \pi}{N} m\right)+2 t^{\prime}\right] d_{m, \alpha}^{\dagger(+)} d_{m, \alpha}^{(+)} \\
& -\sum_{m=1}^{N}\left[2 t \cos \left(\frac{2 \pi}{N} m\right)-2 t^{\prime}\right] d_{m, \alpha}^{\dagger(-)} d_{m, \alpha}^{(-)} \\
& +\frac{4 N}{J} t^{2}+\frac{8 N}{J^{\prime}} t^{\prime 2}
\end{aligned}
$$

This expression clearly represents a decoupled two-band tight-binding model.

The constraint (3), meaning one electron per site, forces the system to be exactly at half filling. Low-energy excitations are then achieved by creating holes just below the Fermi surface and creating electrons just above it. ${ }^{11}$ Notice that this can be done only if

$$
\left|t^{\prime}\right|<|t|
$$

that is, when the Fermi level crosses both bands. If this condition is not satisfied, the system presents a finite-energy gap to spin excitations.

The actual values of $t$ and $t^{\prime}$ are determined by minimizing the energy of Eq. (13). In order to perform this evaluation we introduce a lattice spacing $a$ and a position coordinate $\quad x=n a \quad(x \in[0, L=N a]) ; \quad$ the appropriate pseudomomentum coordinate is $k=m 2 \pi /(\mathrm{Na}) \quad(k$ $\in[-\pi / a, \pi / a])$. The mean-field Hamiltonian then reads

$$
\begin{aligned}
H_{m f}= & -\frac{L}{2 \pi} \int_{-\pi / a}^{\pi / a}\left[2 t \cos (k a)+2 t^{\prime}\right] d_{\alpha}^{\dagger(+)}(k) d_{\alpha}^{(+)}(k) d k \\
& -\frac{L}{2 \pi} \int_{-\pi / a}^{\pi / a}\left[2 t \cos (k a)-2 t^{\prime}\right] d_{\alpha}^{\dagger(-)}(k) d_{\alpha}^{(-)}(k) d k \\
& +\frac{4 L}{a J} t^{2}+\frac{8 L}{a J^{\prime}} t^{\prime 2},
\end{aligned}
$$

from which we can read the dispersion relations for each band, sketched in Fig. 1,

$$
\epsilon^{(+)}(k)=-2 t \cos (k a)-2 t^{\prime},
$$

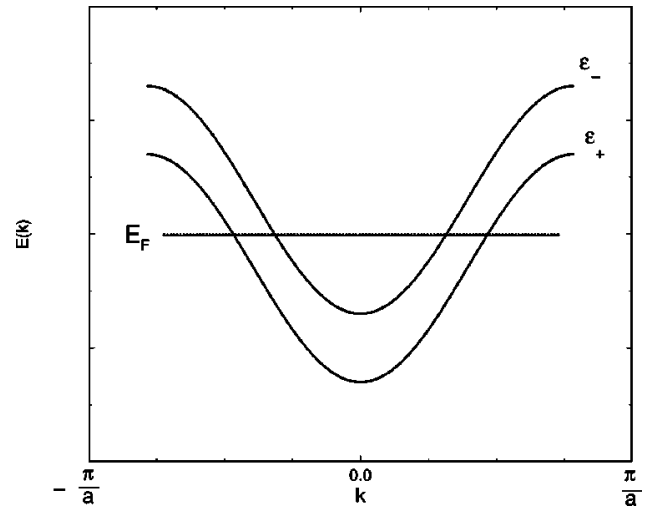

FIG. 1. Dispersion relation for the two-band tight-binding model.

$$
\epsilon^{(-)}(k)=-2 t \cos (k a)+2 t^{\prime}
$$

The Fermi momentum for each band is defined through the equation

$$
\boldsymbol{\epsilon}^{(+)}\left(k_{F}^{(+)}\right)=\epsilon^{(-)}\left(k_{F}^{(-)}\right) .
$$

The local constraint in Eq. (3) leads to the global constraint $N^{(+)}+N^{(-)}=2 N$, where $N^{( \pm)}$is the occupation number operator for each band. Besides, $N^{( \pm)}=(2 N a / \pi) k_{F}^{( \pm)}$. We thus obtain

$$
\begin{gathered}
\cos \left(k_{F}^{(-)} a\right)=\frac{t^{\prime}}{t}=\frac{J^{\prime} B_{0}^{\prime}}{2 J B_{0}}, \\
k_{F}^{(+)}=\pi / a-k_{F}^{(-)},
\end{gathered}
$$

these implying

$$
\boldsymbol{\epsilon}^{(+)}\left(k_{F}^{(+)}\right)=\boldsymbol{\epsilon}^{(-)}\left(k_{F}^{(-)}\right)=0 .
$$

The values of $t$ and $t^{\prime}$ are now determined by minimizing the energy of Eq. (15) under half-filling conditions,

$$
\begin{aligned}
E_{m f}= & -\frac{L}{2 \pi} \int_{-k_{F}^{(+)}}^{k_{F}^{(+)}}\left[2 t \cos (k a)+2 t^{\prime}\right] 2 d k \\
& -\frac{L}{2 \pi} \int_{-k_{F}^{(-)}}^{k_{F}^{(-)}}\left[2 t \cos (k a)-2 t^{\prime}\right] 2 d k+\frac{4 L}{a J} t^{2}+\frac{8 L}{a J^{\prime}} t^{\prime 2} .
\end{aligned}
$$

Notice that $t<0$ just inverts the cosine curves, translating the Brillouin zone considered in $\pi / a$, and $t^{\prime}<0$ would just trade the roles of the two bands. Then, the relevant sector in the $t, t^{\prime}$ plane is $t \geqslant 0$ and $t^{\prime} \geqslant 0$. In this sector, the expression for the energy is

$$
\begin{aligned}
\frac{a \pi}{L} E_{m f}= & -8 t \sin \left(k_{F}^{(-)} a\right)-4 t^{\prime}\left(\pi-2 k_{F}^{(-)} a\right) \\
& +\frac{4 \pi}{J} t^{2}+\frac{8 \pi}{J^{\prime}} t^{\prime 2}, \quad t^{\prime}<t,
\end{aligned}
$$

and 


$$
\frac{a \pi}{L} E_{m f}=-4 \pi t^{\prime}+\frac{4 \pi}{J} t^{2}+\frac{8 \pi}{J^{\prime}} t^{\prime 2}, \quad t^{\prime}>t .
$$

The analysis of the above equations shows that for $J^{\prime}$ $<8 / \pi^{2} J$, the MF configuration depicts two bands that coincide with those corresponding to two decoupled chains ( $t$ $\left.=J / \pi, t^{\prime}=0\right)$, with Fermi momentum $k_{F}=k_{F}^{(-)}=k_{F}^{(+)}$ $=\pi /(2 a)$. Notice that the condition in Eq. (14) holds and the linearization procedure around this minimum is valid.

On the contrary, for $J^{\prime}>\left(8 / \pi^{2}\right) J$, we find that the global energy minimum corresponds to the point $t=0, t^{\prime}=J^{\prime} / 4$ where the condition in Eq. (14) does not hold (notice that there is still another local minimum while $J^{\prime}<2 J$ ). The system in this configuration, that describes the strong coupling phase presents a finite-energy gap to spin excitations.

In the following two sections we will explore the $J^{\prime}$ $<8 / \pi^{2} J$ region.

\section{FLUCTUATIONS AND CONSTRAINTS: THE $S U(4)_{1} / U(1)$ COSET THEORY}

In this section we take the continuum limit of the MF Hamiltonian in Eq. (13) and then include fluctuations around MF and constraints in Eq. (3). The outcome of this procedure is a perturbed $S U(4)_{1} / U(1)$ coset theory.

\section{A. Low-energy linearization in the thermodynamical continuum limit}

In the region we consider $\left(J^{\prime}<8 / \pi^{2} J\right)$ the mean-field dispersion relation consists of two coinciding bands of amplitude $2 J / \pi$. Linearization of low-energy excitations can be done around $k_{F}=\pi /(2 a)$ in the usual way. The bandwidth will limit the validity of the resulting effective field theory to energies much smaller than $J$, independently of $J^{\prime}$.

Low-energy excitations in the thermodynamical continuum limit of the tight-binding model at half filling can be linearized in terms of Dirac fermions. ${ }^{11}$ Fermionic position space operators $c^{( \pm)}$for each band are readily written in terms of Dirac fermions $\psi^{( \pm)}(x)$ as

$$
\begin{aligned}
& c_{n, \alpha}^{(+)}=\sqrt{a}\left[\exp \left(-i k_{F} x\right) \psi_{R, \alpha}^{(+)}(x)+\exp \left(i k_{F} x\right) \psi_{L, \alpha}^{(+)}(x)\right], \\
& c_{n, \alpha}^{(-)}=\sqrt{a}\left[\exp \left(-i k_{F} x\right) \psi_{R, \alpha}^{(-)}(x)+\exp \left(i k_{F} x\right) \psi_{L, \alpha}^{(-)}(x)\right] .
\end{aligned}
$$

Here $\psi_{R, \alpha}^{(+)}$and $\psi_{L, \alpha}^{(+)}$stand for the right and left components of a Dirac spinor $\Psi_{\alpha}^{(+)}$and so on. Dirac gamma matrices are taken as $\gamma_{0}=\sigma_{1}, \gamma_{1}=\sigma_{2}$. Notice that there is a total of four Dirac fermion species; using the notation $\alpha=\uparrow, \downarrow$ they are $(+, \uparrow),(-, \uparrow),(+, \downarrow),(-, \downarrow)$, which will be respectively denoted $\Psi_{i}(x)$ with $i=1,2,3,4$. Summation over repeated fermion-species indices will be understood.

We arrive then at the linearized MF Hamiltonian

$$
H_{m f}=v_{F} \int d x \bar{\Psi}_{i}(x) \gamma_{1} \partial_{x} \Psi_{i}(x)
$$

where

$$
v_{F}=2 \operatorname{ta} \sin \left(k_{F} a\right)=2 J a / \pi
$$

is the Fermi velocity and $\bar{\Psi}_{i}=\Psi_{i}^{\dagger} \gamma_{0}$. All four $\Psi$ fields have the same Fermi velocity, and the model, up to this point, possesses a manifest $U(4)$ symmetry.

\section{B. Fluctuations around mean field}

We include now the fluctuation fields $B_{n}^{(1)}, B_{n}^{(2)}, B_{n}^{\prime}$. As we look for the continuum limit of the Hamiltonian (5), we will keep only relevant powers in $a$, as compared with Eq. (27).

In order to keep track of $a$ orders, it is useful to make explicit the order $a$ contribution of fermion bilinears by defining

$$
\begin{gathered}
z_{n}^{(1)}=a^{-1} c_{n, \alpha}^{\dagger(1)} c_{n+1, \alpha}^{(1)}, \\
z_{n}^{(2)}=a^{-1} c_{n, \alpha}^{\dagger(2)} c_{n+1, \alpha}^{(2)}, \\
z_{n}^{(3)}=a^{-1} c_{n, \alpha}^{\dagger(1)} c_{n, \alpha}^{(2)},
\end{gathered}
$$

so that the leading order for each $z$ is $a^{0}$. Notice that $z^{(1)}$ and $z^{(2)}$ still have to be expanded, as $(n+1) a=x+a$; the only relevant term in this expansion is that linear in $a$, containing first derivatives of $\psi$ fields. Our notation will be

$$
z_{n}^{(i)}=w_{n}^{(i)}+a v_{n}^{(i)}
$$

(notice that $v_{n}^{(3)}=0$ ).

The relevant expansions for the $B$ fields, taking into account that the MF value of $B_{0}^{\prime}$ vanishes, are

$$
B_{n}^{(l)}=B_{0}+i a B_{0} A_{n}^{(l)}+a B_{0} R_{n}^{(l)}+O\left(a^{2}\right), \quad(l=1,2)
$$

$$
B_{n}^{\prime}=i a A_{n}^{\prime}+a R_{n}^{\prime}+O\left(a^{2}\right)
$$

In particular, the terms quadratic in $B$ must be expanded as

$$
\begin{gathered}
B_{n}^{\dagger(l)} B_{n}^{(l)}=B_{0}^{2}+2 a B_{0}^{2} R_{n}^{(l)}+2 a^{2} B_{0}^{2} R_{n}^{(l) 2}, \quad(l=1,2) \\
B_{n}^{\prime \dagger} B_{n}^{\prime}=a^{2} A_{n}^{\prime 2}+a^{2} R_{n}^{\prime 2}
\end{gathered}
$$

Using all of these, and making explicit the sums over $l$ $=1,2$, the effective low-energy Hamiltonian for Eq. (5) is written as

$$
H_{e f f}=H^{(1)}+H^{(2)}+H^{(3)}+O\left(a^{3}\right),
$$

where 


$$
\begin{aligned}
H^{(1)}= & \frac{J}{2} N B_{0}^{2}+\frac{J}{2} \sum_{n=1}^{N} a B_{0}\left(z_{n}^{(1)}+z_{n}^{\dagger(1)}\right) \\
& +\frac{J}{2} \sum_{n=1}^{N} i a^{2} B_{0} A_{n}^{(1)}\left(w_{n}^{(1)}-w_{n}^{\dagger(1)}\right) \\
& +\frac{J}{2} \sum_{n=1}^{N} a\left[a B_{0} R_{n}^{(1)}\left(w_{n}^{(1)}+w_{n}^{\dagger(1)}\right)\right. \\
& \left.+2 B_{0}^{2} R_{n}^{(1)}+2 a B_{0}^{2} R_{n}^{(1) 2}\right] \\
H^{(2)}= & \frac{J}{2} N B_{0}^{2}+\frac{J}{2} \sum_{n=1}^{N} a B_{0}\left(z_{n}^{(2)}+z_{n}^{\dagger(2)}\right) \\
+ & \frac{J}{2} \sum_{n=1}^{N} i a^{2} B_{0} A_{n}^{(2)}\left(w_{n}^{(2)}-w_{n}^{\dagger(2)}\right) \\
+ & \frac{J}{2} \sum_{n=1}^{N} a\left[a B_{0} R_{n}^{(2)}\left(w_{n}^{(2)}+w_{n}^{\dagger(2)}\right)+2 B_{0}^{2} R_{n}^{(2)}\right. \\
+ & \left.2 a B_{0}^{2} R_{n}^{(2) 2}\right], \\
H^{(3)}= & \frac{J^{\prime}}{2} \sum_{n=1}^{N} i a^{2}\left[A_{n}^{\prime}\left(w_{n}^{(3)}-w_{n}^{\dagger(3)}\right)+A_{n}^{\prime 2}\right] \\
& +\frac{J^{\prime}}{2} \sum_{n=1}^{N} a^{2}\left[R_{n}^{\prime}\left(w_{n}^{(3)}+w_{n}^{\dagger(3)}\right)+R_{n}^{\prime 2}\right] .
\end{aligned}
$$

The main things to notice here are:

(i) There are irrelevant (divergent) constant terms. This is expected from the combination of Hubbard-Stratonovich and MF techniques.

(ii) The terms without fluctuations in $H^{(1)}$ and $H^{(2)}$ provide the two decoupled chains MF results discussed in the previous section.

(iii) The $A^{(1)}$ and $A^{(2)}$ fields act as Lagrange multipliers; their total contribution to the effective action in the continuum limit reduces to a term

$$
\begin{aligned}
& -i \frac{v_{F}}{2} \int d x\left\{\left[\bar{\Psi}_{i}(x) \gamma_{1} \Psi_{i}(x)\right]\left[A^{(1)}(x)+A^{(2)}(x)\right]\right. \\
& +\left[\bar{\Psi}_{i}(x) \gamma_{1}\left(\sigma_{1} \otimes 1\right)_{i j} \Psi_{j}(x)\left[A^{(1)}(x)-A^{(2)}(x)\right]\right\} .
\end{aligned}
$$

In the notation of Eq. (37) the first matrix $\left(\sigma_{1}\right)$ refers to isospin indices $(+),(-)$, while the second one (1) refers to spin indices $\uparrow, \downarrow$.

(iv) The presence of a quadratic term in the $A^{\prime}$ field, with proper sign, allows for a trivial Gaussian integration. The same can be done with the $R$ fields. These of course bring back the original spin-spin rung interactions. In the present scheme their contribution includes quadratic terms in the $c$ operators that lead to a redefinition of the Fermi velocity $v_{F} \rightarrow v_{F} / 2$ and quartic perturbations that can be arranged as

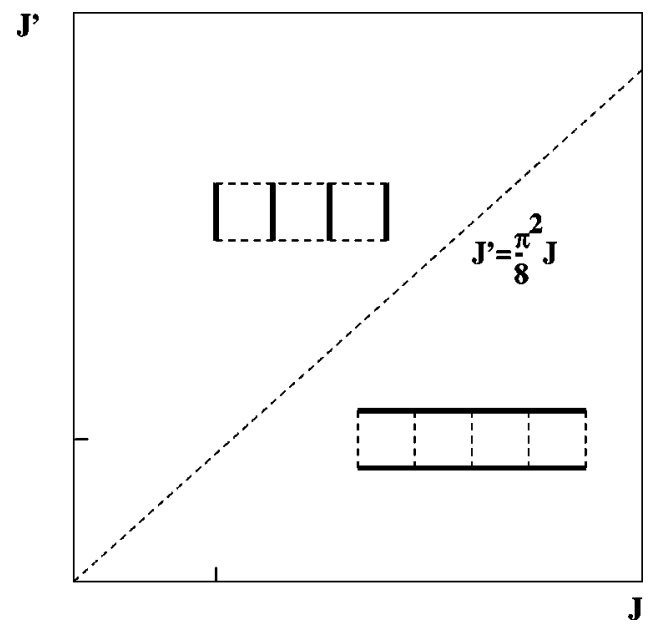

FIG. 2. Phase diagram of the spin ladder. Bold bonds correspond to nonzero links in the MF approximation.

$$
\begin{aligned}
& -\frac{J a^{2}}{16} \sum_{n=1}^{N}\left[\left(w_{n}^{(1)}+w_{n}^{\dagger(1)}\right)^{2}+\left(w_{n}^{(2)}+w_{n}^{\dagger(2)}\right)^{2}\right] \\
& -\frac{J^{\prime} a^{2}}{2} \sum_{n=1}^{N} w_{n}^{(3)} w_{n}^{\dagger(3)} .
\end{aligned}
$$

The continuum form of these quartic perturbations in terms of Dirac fermions is lengthy. We will write them down below, after introducing a convenient notation.

We notice that for $J^{\prime}<8 / \pi^{2} J$, our approach leads to a description of the system that is the same as the one obtained in perturbative treatments, in principle valid for $J^{\prime} \ll J^{4,12}$ In particular, the first two terms in Eq. (38) give rise to the well-known marginally irrelevant perturbation terms in the individual chains. However, our approach does not rely on any perturbative treatment of $J^{\prime}$ and in particular allows for the determination of the phase diagram of the system, i.e., it predicts a critical value of the ratio $J^{\prime} / J$ that separates the two different regimes in the two-leg ladder. The situation is depicted in Fig. 2.

Moreover, we show in the next section that the weakcoupling structure unraveled in Ref. 4 arises naturally within our approach.

\section{Constraints}

In this section we express the constraints (3) in terms of the linearized fermion fields and discuss how to implement them in the evaluation of the partition function for the spin ladder.

In the continuum limit the constraint on the occupation number at each site $(l), n$ separates in four parts, corresponding to oscillating and nonoscillating terms associated to each band. They read

$$
\bar{\Psi}_{i} \gamma_{0} \Psi_{i}=\text { const, }
$$

which, implemented through a Lagrange multiplier $a_{0}$, provides the time component of a gauge field implementing a diagonal $U(1)$ coset constraint $a_{\mu}=\left(a_{0}, A^{(1)}+A^{(2)}\right)$; 


$$
\bar{\Psi}_{i} \gamma_{0}\left(\sigma_{1} \otimes l\right)_{i j} \Psi_{j}=0,
$$

which, implemented through a Lagrange multiplier $b_{0}$, provides the time component of a second gauge field implementing the isospin $U(1)$ coset constraint $b_{\mu}=\left(b_{0}, A^{(1)}\right.$ $-A(2))$. These last two constraints

$$
\begin{gathered}
\bar{\Psi}_{i} \Psi_{i}=0, \\
\bar{\Psi}_{i}\left(\sigma_{1} \otimes 1\right)_{i j} \Psi_{j}=0
\end{gathered}
$$

lead to marginally irrelevant quartic perturbation terms when implemented through

$$
\delta(\mathcal{O}(x)) \propto \lim _{\eta \rightarrow \infty} e^{-\eta \mathcal{O}^{\dagger}(x) \mathcal{O}(x)},
$$

just as in the case of decoupled chains.

To conclude this section, we collect all the terms in the effective low-energy Hamiltonian, which finally reads

$$
\begin{aligned}
H_{e f f}= & \frac{a J}{\pi} \int d x \bar{\Psi}_{i}(x)\left[\left(\gamma_{1} \partial_{x}-i \gamma_{\mu} a_{\mu}\right) \delta_{i j}\right. \\
& \left.-i \gamma_{\mu}\left(\sigma_{1} \otimes 1\right)_{i j} b_{\mu}\right] \Psi_{j}(x)+\Delta H_{e f f},
\end{aligned}
$$

where $\Delta H_{\text {eff }}$ includes quartic terms in fermionic fields that arise from Eqs. (38), (41), and (42).

Clearly, the unperturbed theory possesses a $U(4)$ symmetry that is gauged by a diagonal $U(1)$ field $\left(a_{\mu}\right)$ and an isospin $U(1)$ field $\left(b_{\mu}\right)$, which leads to the coset

$$
\frac{U(4)}{U(1)_{\text {diag }} \times U(1)_{\text {iso }}}=\frac{S U(4)_{1}}{U(1)_{\text {iso }}},
$$

where $S U(4)_{1}$ stands for the level $k=1$ Wess-ZuminoWitten (WZW) theory. ${ }^{13,14}$

Before displaying the explicit expression for the perturbations it is worth discussing in more detail the coset structure of the quadratic part of the Hamiltonian.

\section{IV. $S U(2)_{2} \times Z_{2}$ EMBEDDING-THE PERTURBATIONS IN A NEW LANGUAGE}

As it is known, the coset CFT $S U(4)_{1}$ can be alternatively described through the embedding ${ }^{15}$

$$
S U(4)_{1}=S U(2)_{2} \times S U(2)_{2} .
$$

The conformal central charges of the two theories coincide and primary fields in the $S U(4)_{1}$ can be written in terms of primaries in the two $S U(2)_{2}$ sectors. This will presently prove to be useful in the treatment of the perturbations. The different $S U(2)_{2}$ sectors in this embedding are naturally identified in Eq. (44) as the spin and isospin sectors, by virtue of the $\sigma_{1} \otimes 1$ nondiagonal structure. Moreover, in this language the second Lagrange multiplier $b_{\mu}$ gauges a $U(1)$ subgroup of the isospin $S U(2)_{2}$ sector giving rise to

$$
S U(2)_{2}^{\text {spin }} \times S U(2)_{2}^{i \text { sospin }} / U(1)^{i \text { sospin }}=S U(2)_{2}^{\text {spin }} \times Z_{2} .
$$

The last factor has been identified in Ref. 4 from the $Z_{2}$ structure of a two-chain system.
All of this is most easily shown in the bosonized version of the coset CFT. To this end we write fermion bilinears as ${ }^{13,16}$

$$
\psi_{R}^{\dagger} i \psi_{L}^{\bar{j}}=M \Phi_{\square}^{i \bar{j}},
$$

where $M$ is a renormalization constant, and we have introduced bar indices in order to distinguish components transforming in the right and left fundamental representations of $S U(4)_{1}$. The $\square$ subindex indicates the fundamental representation in the standard Young tableaux notation. In identifying the two $S U(2)_{2}$ sectors we find it is useful to keep the original spin and isospin (band) indices, writing

$$
\Phi_{\square}^{i \bar{j}}=\Phi_{\square}^{a \alpha, \overline{b \beta}},
$$

where we now use $a, \bar{b}$ for $(+),(-)$.

This field $\Phi^{i j}$ has scaling dimension $\frac{3}{4}$ and its components can be written in terms of products of the components of the fields in the fundamental representations of the two $S U(2)_{2}$ sectors as

$$
\Phi_{\square}^{a \alpha, \overline{b \beta}}=\phi_{\square}^{a, \bar{b}} \phi_{\square}^{\prime \alpha, \bar{\beta}},
$$

where $\phi$ and $\phi^{\prime}$ are the primary fields in the fundamental (spin- $\frac{1}{2}$ ) representation of the two $S U(2)_{2}$ isospin and spin sectors, respectively. These fields have scaling dimension $\frac{3}{8}$ so the product has the right dimension $\frac{3}{4}$ and, moreover, correlation functions of the fields on both sides coincide.

The other primary field in the $S U(4)_{1}$ CFT is the one transforming in the antisymmetric $(6 \times \overline{6})$ representation, which in the Young tableaux notation should read $\Phi_{\square}$. It is built up from the antisymmetric product of two fields in the fundamental representation

$$
\Phi_{\boxminus}=\mathcal{A}\left(\Phi_{\square} \Phi_{\square}\right) .
$$

This field has scaling dimension 1 and can be mapped into $S U(2)_{2}$ fields as

$$
\begin{aligned}
& \Phi_{\boxminus}^{\left[\left(a_{1} \alpha_{1}\right),\left(a_{2}, \alpha_{2}\right)\right],\left[\left(\overline{b_{1}} \overline{\beta_{1}}\right),\left(\overline{b_{2}}, \overline{\beta_{2}}\right)\right]} \\
& =\phi_{\square}^{\left\{a_{1} a_{2}\right\},\left\{\overline{b_{1}} \bar{b}_{2}\right\}} \boldsymbol{\epsilon}^{\alpha_{1} \alpha_{2}} \boldsymbol{\epsilon}^{\overline{\beta_{1}} \bar{\beta}_{2}}+\boldsymbol{\epsilon}^{a_{1} a_{2}} \boldsymbol{\epsilon}^{\overline{b_{1}} \bar{b}_{2}} \boldsymbol{\phi}_{\square}^{\prime\left\{\alpha_{1} \alpha_{2}\right\}\left\{\overline{\beta_{1}} \overline{\beta_{2}}\right\}},
\end{aligned}
$$

where $\phi_{\square}, \phi_{\square}^{\prime}$ are the primary fields in the symmetric (spin-1) representations of the two $S U(2)_{2}$ sectors that have the correct scaling dimension 1. In Eq. (52) we have used the symbols $\{$,$\} and [$,$] to indicate, respectively, symmetrization$ and antisymmetrization of indices.

We are now ready to analyze the different perturbation terms in $\Delta H_{e f f}$. First of all, contributions coming from intrachain couplings and constraints are known to be marginally irrelevant, just as in the case of decoupled chains. ${ }^{8}$

The interchain perturbation terms in $\Delta H_{\text {eff }}$ [those arising from the last term in Eq. (38)] can be separated into two groups according to their scaling dimensions: there are terms that correspond to relevant operators (scaling dimension 1) that can be identified with certain linear combinations of the 
components of the primary (51) in the coset theory (44), and current-current terms, which have scaling dimension 2 and are hence marginal.

More precisely, for the relevant part we can write

$$
\text { relevant perturbations }=-\lambda \int d x\left[\operatorname{Tr}\left(A \Phi_{\boxminus}\right)+\text { H.c. }\right] \text {, }
$$

where $\lambda \propto J^{\prime}$ and $A$ is given by

$$
A=\left(\begin{array}{cccccc}
-1 & 0 & 0 & 0 & 0 & 0 \\
0 & \frac{1}{2} & 0 & 0 & -\frac{1}{2} & 0 \\
0 & 0 & -\frac{1}{2} & \frac{1}{2} & 0 & 0 \\
0 & 0 & \frac{1}{2} & -\frac{1}{2} & 0 & 0 \\
0 & -\frac{1}{2} & 0 & 0 & \frac{1}{2} & 0 \\
0 & 0 & 0 & 0 & 0 & -1
\end{array}\right)
$$

(see the appendix for details). Using the identifications described above and after some straightforward algebra we can readily identify the perturbation terms (53) in the embedding theory as

$$
\begin{aligned}
\text { relevant perturbations }= & -\lambda \int d x \operatorname{Tr}\left(\phi_{\square}+\text { H.c. }\right) \\
& +\frac{\lambda}{2} \int d x \operatorname{Tr}\left(\phi_{\square}^{\prime} \sigma_{1} \phi_{\square}^{\prime \dagger} \sigma_{1}+\text { H.c. }\right) .
\end{aligned}
$$

To analyze the effect of these perturbation terms it is convenient to reformulate the $S U(2)_{2}$ WZW sector in terms of three decoupled Majorana fermions, and in this new language it is easy to see that the first term gives a mass to all three Majorana fields. ${ }^{17}$ The second one is simply the energy operator of the remaining Majorana sector. ${ }^{14,18}$ As they are all perturbations of dimension 1 we see that the gap opens linearly with the interchain coupling as predicted from the weak-coupling limit. ${ }^{3,4}$ Note the different sign in the masses of the two sectors, also in agreement with the weak-coupling analysis.

As for the current-current terms, they correspond to marginal perturbations and can be written as

marginal perturbations

$$
\begin{aligned}
= & -\frac{a J^{\prime}}{8} \int d x\left\{-\psi_{L}^{\dagger}\left[\left(\sigma_{3}+i \sigma_{2}\right) \otimes 1\right] \psi_{L} \psi_{L}^{\dagger}\left[\left(\sigma_{3}-i \sigma_{2}\right)\right.\right. \\
& \left.\otimes 1] \psi_{L}-R \leftrightarrow L\right\}-\frac{a J^{\prime}}{8} \int d x\left\{\psi _ { L } ^ { \dagger } \left[\left(\sigma_{3}+i \sigma_{2}\right)\right.\right. \\
& \left.\otimes 1] \psi_{L} \psi_{R}^{\dagger}\left[\left(\sigma_{3}-i \sigma_{2}\right) \otimes 1\right] \psi_{R}+R \leftrightarrow L\right\} \\
& -\frac{a J^{\prime}}{8} \int d x\left[-\psi_{L}^{\dagger}(1 \otimes 1) \psi_{L} \psi_{R}^{\dagger}(1 \otimes 1) \psi_{R}\right.
\end{aligned}
$$

$$
\begin{aligned}
& \left.-\psi_{L}^{\dagger}(1 \otimes \vec{\sigma}) \psi_{L} \cdot \psi_{R}^{\dagger}(1 \otimes \vec{\sigma}) \psi_{R}\right]-\frac{a J^{\prime}}{8} \int d x \\
& \times\left[\psi_{L}^{\dagger}\left(\sigma_{1} \otimes 1\right) \psi_{L} \psi_{R}^{\dagger}\left(\sigma_{1} \otimes 1\right) \psi_{R}\right. \\
& \left.+\psi_{L}^{\dagger}\left(\sigma_{1} \otimes \vec{\sigma}\right) \psi_{L} \cdot \psi_{R}^{\dagger}\left(\sigma_{1} \otimes \vec{\sigma}\right) \psi_{R}\right] .
\end{aligned}
$$

The first two terms (first line) renormalize the Fermi velocity of the Majorana (isospin) sector, while the third and fourth (second line) corresponds to marginal forward-scattering terms in the same sector. The fifth and seventh terms are effectively zero due to the constraints on the two corresponding $U(1)$ currents and the sixth term correspond to the marginal forward-scattering terms in the spin sector. The very last one mixes spin and isospin sectors. This last contribution is nevertheless marginal, so it does not change the lowenergy physics, which in the present case is dominated by the relevant perturbations already discussed. Its effect could be important in the analysis of, e.g., zigzag ladders where the relevant perturbations are wiped out, as we show in the next section, and only marginal interactions play a role. ${ }^{19-22}$

It can be easily shown that the marginal terms present on each separate chain, written in the present language, correspond to the sixth and eighth terms in the above expression. Due to the fact that these terms correspond to marginally irrelevant couplings and that they form a closed algebra, they will have no effect on the low energy dynamics whatsoever. After having observed that, one can see that the effective theory consists of two sectors that are decoupled from each other.

\section{OTHER STRUCTURES: CROSSED AND ZIGZAG LADDERS}

In this section we will extend our previous analysis to more general situations, which are not only of academic interest, but are relevant in the analysis of real materials. These more general situations arise when other (diagonal) couplings between spins in neighboring chains are not negligible. The two structures that we analyze now are the socalled crossed ladders, ${ }^{23,25}$ in which couplings along the two diagonals are added, and zigzag ladders, in which only one diagonal coupling is added. ${ }^{19-22}$ Another potential application of the present formalism would be the study of the interplay between interchain coupling and dimerization along the legs. ${ }^{26-28}$

\section{A. Crossed ladders}

We consider a Heisenberg Hamiltonian given by

$$
\begin{aligned}
H= & \sum_{n=1}^{N} \sum_{l=1}^{2} J \vec{S}_{n}^{(l)} \cdot \vec{S}_{n+1}^{(l)}+J^{\prime} \sum_{n=1}^{N} \vec{S}_{n}^{(1)} \cdot \vec{S}_{n}^{(2)} \\
& +J_{\times} \sum_{n=1}^{N}\left(\vec{S}_{n}^{(1)} \cdot \vec{S}_{n+1}^{(2)}+\vec{S}_{n+1}^{(1)} \cdot \vec{S}_{n}^{(2)}\right)
\end{aligned}
$$

where the last term corresponds to additional diagonal couplings. 


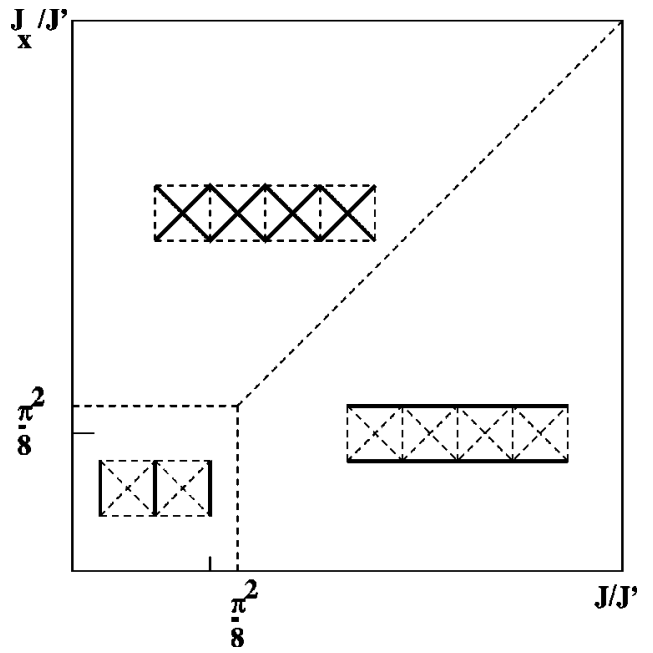

FIG. 3. Phase diagram of the crossed ladder. Bold bonds correspond again to nonzero links in the MF approximation.

Following the same approach as for the normal ladder we introduce Hubbard-Stratonovich fields associated to each coupling and perform a three-parameter MF analysis proposing constant values for the intrachain, the interchain (rung), and the interchain (diagonal) couplings. We find two different regions in the parameter space $\left(J / J^{\prime}, J_{\times} / J^{\prime}\right)$. It should be noted that this Hamiltonian is dual under the interchange $J \leftrightarrow J_{\times}$; then it is enough to study the region $J_{\times} \leqslant J$. (See Fig. 3.)

(i) If $J / J^{\prime}>\pi^{2} / 8$, the MF analysis yields the system in a weak-coupling regime, and following all the same steps as before, we arrive at the same effective-field theory with the noticeable change that the coefficient of the relevant perturbations is now shifted as $J^{\prime}-2 J_{\times}$. As in the weak-coupling analysis, ${ }^{23,24}$ one immediately sees that there is a line in which the relevant perturbations vanish. On this line one could expect a massless regime, as suggested by numerical studies. $^{23,25}$ However in a recent treatment of the resulting bosonized Hamiltonian it was shown that the current-current terms are marginally relevant and a gap opens. ${ }^{24}$ The same conclusion is attained in our resulting effective theory. Again, the new feature here is that we find the region of validity of the weak-coupling effective-field theory to go up to $J^{\prime}=8 / \pi^{2} J$.

(ii) If $J / J^{\prime}<\pi^{2} / 8$, the system falls in a strong-coupling regime in which the two dispersion bands are separated by a gap $\left(\propto J^{\prime}\right)$ and then a low-energy effective-field theory description is not suitable here.

\section{B. Zigzag ladders}

The Hamiltonian is given by

$$
H=\sum_{n=1}^{N} \sum_{l=1}^{2} J \vec{S}_{n}^{(l)} \cdot \vec{S}_{n+1}^{(l)}+J^{\prime} \sum_{n=1}^{N} \vec{S}_{n}^{(1)} \cdot\left(\vec{S}_{n}^{(2)}+\vec{S}_{n+1}^{(2)}\right) .
$$

Introducing again Hubbard-Stratonovich fields associated to each coupling and performing a MF analysis with constant values for the intrachain and interchain couplings, we find a

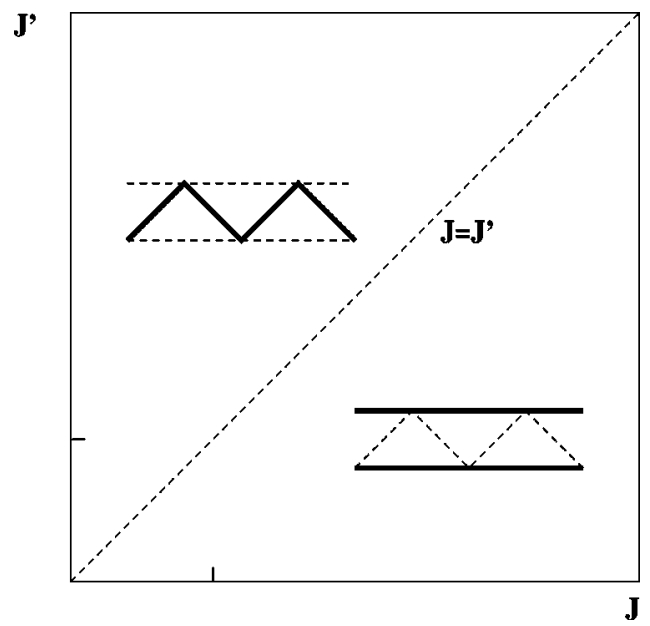

FIG. 4. Phase diagram of the zigzag ladder. Bold bonds correspond again to nonzero links in the MF approximation.

different situation: while we still find a regime, which now exists for $J^{\prime}<J$, in which we reobtain the standard weakcoupling results, we find that the "strong-coupling" regime $\left(J^{\prime}>J\right)$ can still be described by an effective low-energy field theory. (See Fig. 4.)

More precisely, in the regime in which $J^{\prime}<J$ we find that all relevant perturbations cancel in a way similar to that found in the weak-coupling limit. ${ }^{19-22}$ The effective lowenergy theory corresponds to the same coset theory, perturbed only by the operators appearing in the first, third, and fourth lines in Eq. (56). The so-called parity breaking terms first studied in Ref. 21 appear in the present approach from the next-to-leading order in the lattice spacing $a$ in the expansion of the modified version of Eq. (38).

In the other regime $\left(J^{\prime}>J\right)$, the bands at the MF minimum are given by

$$
\begin{gathered}
\epsilon^{(+)}(k)=-\frac{J^{\prime}}{\pi} \sqrt{2[1+\cos (k a)]}, \\
\epsilon^{(-)}(k)=\frac{J^{\prime}}{\pi} \sqrt{2[1+\cos (k a)]},
\end{gathered}
$$

there being no gap between them, and a field-theory description is still possible. The difference is that the low-energy effective theory should in this case be built up on only two fermion species, exhibiting $S U(2)_{1}$ symmetry. See Fig. 5. This should correspond to the description of a single chain plus next-nearest-neighbor interactions, which is the suitable picture for the regime where $J^{\prime}$ dominates.

Once again, our method allows for the construction of an effective-field theory for the full range of couplings and in particular allows us to study the transition from the massless ( $c=1$ ) $J=0$ limit to the massive Kosterlitz-Thouless regime known to arise at $J \approx 0.24 J^{\prime},{ }^{29}$ which should, according to our analysis, extend to the limit $J^{\prime} \rightarrow 0$.

Since the main purpose of the present paper is to emphasize the potential applications of our approach, the analysis of these effective-field theories will be addressed in a separate publication. 


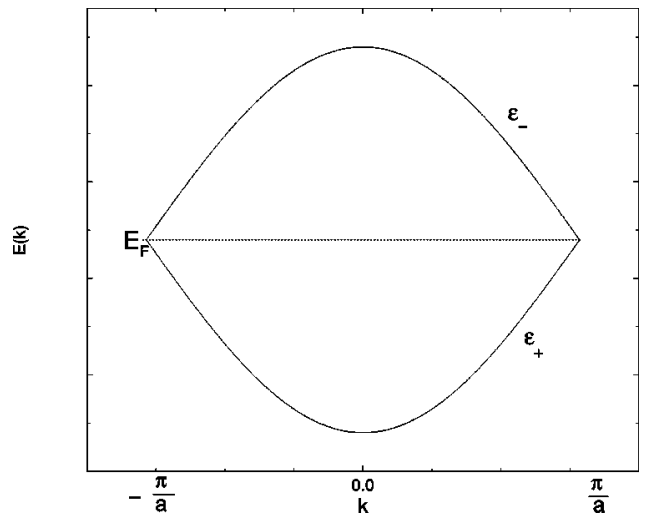

FIG. 5. Energy bands for the zigzag ladder at $J^{\prime}>J \mathrm{MF}$ minimum.

\section{CONCLUSIONS}

The approach developed in the present paper shows that the spectrum predicted from weak-coupling approximation extends up to a finite value of $J^{\prime} / J$, our estimation of this critical value being $J_{c}^{\prime} / J \sim 8 / \pi^{2}$. Beyond this value our MF analysis in Sec. II predicts a crossover to the strong-coupling regime, where the rungs of the ladder become disconnected among them. Fluctuations over this state will restore connectivity and the strong-coupling approach of Refs. 30 and 31 would be the appropriate starting point in this parameter regime. As the classical potential analyzed in Sec. II has a double-well structure in the intermediate region $\left(J^{\prime} \sim J_{c}^{\prime}\right)$ we expect a smooth crossover from a weak- to a strong-coupling regime. Experimental observation of this crossover supposes the variation of the ratio of the exchange parameter. This could in principle be achieved by applying pressure in the perpendicular direction of the ladder axis.

Though our approach starts from a MF analysis, fluctuations are taken into account for all orders. Besides, it allows a classification of all the perturbations in the language of the embedding of the theory into $S U(2)_{2} \times Z_{2}$. One interesting observation that arises is that only the $Z_{2}$ Majorana Fermi velocity is renormalized to first order in $J^{\prime}$ by the interactions.

The study of hole doped spin ladders is a natural extension of our approach. For this case the $t-J$ model should be considered and the charge sector of the theory could be represented by a spinless boson (the slave boson representation). However the magnetic excitations will evolve from the triplet and the singlet found in this paper. The question of the hole pairing due to these excitations could therefore be addressed within our formalism. This will be reported elsewhere.

\section{ACKNOWLEDGMENTS}

We are grateful to E. F. Moreno for useful discussions and computational help. We also thank A. Greco, A. Honecker, A. A. Nersesyan, and P. Pujol for useful comments. We thank CONICET and Fundación Antorchas (Grants No. A-13622/1-106 and A-13740/1-64) for partial support.

\section{APPENDIX}

We write in this appendix the explicit form of some lengthy expressions appearing with compact notation in the main text.

The relevant part of the third term in Eq. (38), appearing in Eq. (44), reads in the continuum limit

$$
\begin{aligned}
\text { relevant perturbations }= & -\frac{J^{\prime} a}{2} \int d x\left(-\Psi_{R, \alpha}^{\dagger(-)} \Psi_{R, \beta}^{\dagger(-)} \Psi_{L, \alpha}^{(-)} \Psi_{L, \beta}^{(-)}+\Psi_{R, \alpha}^{\dagger(-)} \Psi_{R, \beta}^{\dagger(-)} \Psi_{L, \alpha}^{(-)} \Psi_{L, \beta}^{(+)}-\Psi_{R, \alpha}^{\dagger(-)} \Psi_{R, \beta}^{\dagger(-)} \Psi_{L, \alpha}^{(+)} \Psi_{L, \beta}^{(-)}\right. \\
& +\Psi_{R, \alpha}^{\dagger(-)} \Psi_{R, \beta}^{\dagger(-)} \Psi_{L, \alpha}^{(+)} \Psi_{L, \beta}^{(+)}-\Psi_{R, \alpha}^{\dagger(-)} \Psi_{R, \beta}^{\dagger(+)} \Psi_{L, \alpha}^{(-)} \Psi_{L, \beta}^{(-)}+\Psi_{R, \alpha}^{\dagger(-)} \Psi_{R, \beta}^{\dagger(+)} \Psi_{L, \alpha}^{(-)} \Psi_{L, \beta}^{(+)} \\
& -\Psi_{R, \alpha}^{\dagger(-)} \Psi_{R, \beta}^{\dagger(+)} \Psi_{L, \alpha}^{(+)} \Psi_{L, \beta}^{(-)}+\Psi_{R, \alpha}^{\dagger(-)} \Psi_{R, \beta}^{\dagger(+)} \Psi_{L, \alpha}^{(+)} \Psi_{L, \beta}^{(+)}+\Psi_{R, \alpha}^{\dagger(+)} \Psi_{R, \beta}^{\dagger(-)} \Psi_{L, \alpha}^{(-)} \Psi_{L, \beta}^{(-)}-\Psi_{R, \alpha}^{\dagger(+)} \Psi_{R, \beta}^{\dagger(-)} \Psi_{L, \alpha}^{(-)} \Psi_{L, \beta}^{(+)} \\
& +\Psi_{R, \alpha}^{\dagger(+)} \Psi_{R, \beta}^{\dagger(-)} \Psi_{L, \alpha}^{(+)} \Psi_{L, \beta}^{(-)}-\Psi_{R, \alpha}^{\dagger(+)} \Psi_{R, \beta}^{\dagger(-)} \Psi_{L, \alpha}^{(+)} \Psi_{L, \beta}^{(+)}+\Psi_{R, \alpha}^{\dagger(+)} \Psi_{R, \beta}^{\dagger(+)} \Psi_{L, \alpha}^{(-)} \Psi_{L, \beta}^{(-)}-\Psi_{R, \alpha}^{\dagger(+)} \Psi_{R, \beta}^{\dagger(+)} \Psi_{L, \alpha}^{(-)} \Psi_{L, \beta}^{(+)} \\
& +\Psi_{R, \alpha}^{\dagger(+)} \Psi_{R, \beta}^{\dagger(+)} \Psi_{L, \alpha}^{(+)} \Psi_{L, \beta}^{(-)}-\Psi_{R, \alpha}^{\dagger(+)} \Psi_{R, \beta}^{\dagger(+)} \Psi_{L, \alpha}^{(+)} \Psi_{L, \beta}^{(+)}+\text {H.c.), }
\end{aligned}
$$

by simple use of Eqs. (25), (26), (29), and (30).

The explicit form of Eq. (51) in terms of fermions, using Eq. (48), is

$$
\Phi_{\boxminus}^{\left[\left(i_{1} \alpha_{1}\right),\left(i_{2}, \alpha_{2}\right)\right],\left[\left(\overline{j_{1}} \overline{\beta_{1}}\right),\left(\overline{j_{2}}, \overline{\beta_{2}}\right)\right]}=\mathcal{A}\left(\Phi_{\square}^{\left(i_{1} \alpha_{1}\right),\left(\overline{j_{1}} \overline{\beta_{1}}\right)} \Phi_{\square}^{\left(i_{2}, \alpha_{2}\right),\left(\overline{j_{2}}, \overline{\beta_{2}}\right)}\right),
$$

where antisymmetrization affects barred and unbarred pairs of indices separately.

Using Eqs. (A1) and (A2), expression (53) follows immediately. The base used for writing the matrix $A$ in Eq. (54) is the one made explicit with indices in the left-hand side of Eq. (52), ordered as $[(+, \uparrow),(-, \uparrow)],[(+, \uparrow),(+, \downarrow)]$, $[(+, \uparrow),(-, \downarrow)],[(-, \uparrow),(+, \downarrow)],[(-, \uparrow),(-, \downarrow)],[(+, \downarrow),(-, \downarrow)]$. 
${ }^{1}$ For a recent review on the status of experimental results on ladders see E. Dagotto, Rep. Prog. Phys. 62, 1525 (1999).

${ }^{2}$ T. Barnes, E. Dagotto, J. Riera, and E.S. Swanson, Phys. Rev. B 47, 3196 (1993).

${ }^{3}$ K. Totsuka and M. Suzuki, J. Phys.: Condens. Matter 7, 6079 (1995).

${ }^{4}$ D.G. Shelton, A.A. Nersesyan, and A.M. Tsvelik, Phys. Rev. B 53, 8521 (1996).

${ }^{5}$ E. Orignac and R. Citro, Phys. Rev. B 62, 8622 (2000).

${ }^{6}$ M.V. Abrashev, C. Thomsen, and M. Surtchev, Physica C 280, 297 (1997).

${ }^{7}$ J. Marston and I. Affleck, Phys. Rev. B 39, 11538 (1989).

${ }^{8}$ C. Itoi and H. Mukaida, J. Phys. A 27, 4695 (1994).

${ }^{9}$ C. Mudry and E. Fradkin, Phys. Rev. B 50, 11409 (1994).

${ }^{10}$ P.J. Freitas and R.R.P. Singh, Phys. Rev. B 62, 5525 (2000).

11 I. Affleck, in Fields, Strings and Critical Phenomena, Les Houches, Session XLIX, edited by E. Brezin and J. Zinn-Justin (North-Holland, Amsterdam, 1988).

${ }^{12}$ Y. Hosotani, J. Phys. A 30, L757 (1997); 31, 7415(E) (1998).

${ }^{13}$ E. Witten, Commun. Math. Phys. 94, 455 (1984).

${ }^{14}$ V.G. Knizknik and A.B. Zamolodchikov, Nucl. Phys. B 247, 83 (1984).

${ }^{15}$ See, e.g., P. Bouwknegt and K. Schoutens, Phys. Rep. 223, 183 (1993).
${ }^{16}$ S.G. Naculich and H.J. Schnitzer, Nucl. Phys. B 332, 583 (1990).

${ }^{17}$ A.B. Zamolodchikov and V.A. Fateev, Zh. Éksp. Teor. Fiz. 89, 380 (1985) [Sov. Phys. JETP 62, 215 (1985)].

${ }^{18}$ D.C. Cabra and E.F. Moreno, Nucl. Phys. B 475, 522 (1996).

${ }^{19}$ S.R. White and I. Affleck, Phys. Rev. B 54, 9862 (1996).

${ }^{20}$ D. Allen and D. Sénéchal, Phys. Rev. B 55, 299 (1997).

${ }^{21}$ A.A. Nersesyan, A.O. Gogolin, and F.H.L. Essler, Phys. Rev. Lett. 81, 910 (1998).

${ }^{22}$ D.C. Cabra, A. Honecker, and P. Pujol, Eur. Phys. J. B 13, 55 (2000).

${ }^{23}$ Z. Weihong, V. Kotov, and J. Oitmaa, Phys. Rev. B 57, 11439 (1998).

${ }^{24}$ D. Allen, F.H.L. Essler, and A.A. Nersesyan, Phys. Rev. B 61, 8871 (2000).

${ }^{25}$ X. Wang, cond-mat/9803290 (unpublished).

${ }^{26}$ M.A. Martín-Delgado, R. Shankar, and G. Sierra, Phys. Rev. Lett. 77, 3443 (1996).

${ }^{27}$ D.C. Cabra and M.D. Grynberg, Phys. Rev. Lett. 82, 1768 (1999).

${ }^{28}$ Y.-J. Wang and A.A. Nersesyan, Nucl. Phys. B 583, 671 (2000).

${ }^{29}$ S. Eggert, Phys. Rev. B 54, R9612 (1996).

${ }^{30}$ S. Sachdev and R.N. Bhatt, Phys. Rev. B 41, 9323 (1990).

${ }^{31}$ S. Gopalan, T.M. Rice, and M. Sigrist, Phys. Rev. B 49, 8901 (1996). 